\title{
RELIABILITY ANALYSIS OF BEARING CAPACITY OF SQUARE FOOTING ON SOIL WITH STRENGTH ANISOTROPY DUE TO LAYERED MICROSTRUCTURE
}

\author{
MAREK KAWA \\ Wrocław University of Technology, Wrocław, Poland. E-mail: marek.kawa@pwr.edu.pl
}

\begin{abstract}
The paper deals with reliability analysis of square footing on soil with strength anisotropy. The strength of the soil has been described with identified anisotropic strength criterion dedicated to geomaterials with layered microstructure. The analysis assumes dip angle $\alpha$ and azimuth angle $\beta$ which define direction of lamination of the structure to be random variables with given probability density functions. Bearing capacity being a function of these variables is approximated based on results of deterministic simulations obtained for variety of orientations. The weighted regression method by Kaymaz and McMahon within the framework of Response Surface Method is used for the approximation. As a result of analysis, global factor of safety that corresponds to assumed value of probability of failure is determined. The value of the safety factor denotes the ratio between the value of the design load and the mean value of bearing capacity which is needed to reduce the probability of failure to the acceptable level. The procedure of calculating the factor has been presented for two different cases. In the first case, no information about lamination direction of the soil has been provided and thus all the orientations are assumed to be equally probable (uniform distribution). In the second case, statistical information including mean, variance and assumed probability distribution for both $\alpha$ and $\beta$ angle is known. For the latter case, using results obtained for few different values of mean of angle $\alpha$, also the influence of strength anisotropy on the value of global factor of safety is shown.
\end{abstract}

Key words: layered microstructure, square footing, strength anisotropy, reliability analysis, probability of failure, factor of safety

\section{INTRODUCTION}

In many geomaterials a specific pattern can be observed: two or more constituents occur in the form of thin, periodically repeating layers. Sedimentary rocks and soils: schist, shale and varved clay are typical examples of such media. Since dimensions of the layers are usually several times smaller than dimensions of the engineering construction located on this material (for example, width of foundation) these types of media are often referred to as micro-layered. One of the main consequences of layered microstructure occurring in these materials is the strong anisotropy of their strength.

The strength anisotropy, although often omitted, is a very important factor in geotechnical design. In many cases it has strong impact, for instance, on the bearing capacity of footing located on anisotropic soil and thus it should be taken into account in the process of design.

In recent few decades modeling of strength anisotropy has strongly developed. A brief overview of a number of phenomenological anisotropic strength criteria for geomaterials with layered microstructure can be found, e.g., in the work by Duveau et al. [2). In more recent work by Kawa and Łydżba [7] the criterion dedicated to geomaterials with layered microstructure has been identified. The criterion is in fact a conjunction of Pariseau [9] anisotropic criterion and Jaeger [6] critical plane approach. As has been shown, the identified criterion is in a very good agreement with failure surface obtained with micromechanical approach for layered microstructure with strength of constituents governed by the Drucker -Prager criterion.

In the case of anisotropy, the orientation of material directions is a particularly important factor. Geological studies are always subjected to errors and inaccuracies. These errors also apply to the measured values of both dip and azimuth angle, which orients lamination direction in geomaterials with, layered microstructure. Regarding this, designing geotechnical construction located on material described with anisotropic strength criterion, which is already a complex problem, should be performed with particular caution. Some additional safety factor taking into account the 
possible errors in orientation of material directions should be used in the analysis. To calculate value of this safety factor probability theory needs to be employed.

Probabilistic modeling of geomaterials has recently been the subject of interest of many researchers. Reliability approach for design of bearing capacity of strip footing on anisotropic soil has been presented, for example, in the work by Vessia et al. [11]. In this work, the analysis has been conducted using random field theory. The material directions however have been assumed a priori as horizontal and vertical ones and thus the bearing capacity does not depend on their orientation. Another approach is presented in work by Brząkała [1], where problem of uniaxial compression strength for rock with the set of random joints is considered. In this work orientation of the joints has been assumed random variable. The critical load of the rock in the case considered can however be obtained in the close analytical form which strongly simplifies the analysis.

In the present paper, the reliability analysis of bearing capacity of square footing on micro-layered soil is conducted. The orientation of stratification direction plays the main role in problem formulation. The probabilistic modeling is based on deterministic numerical calculations (Finite Difference Method) of bearing capacity performed for variety of orientations. Using these results together with classical tools of structural reliability as Response Surface Method and Monte Carlo Simulation the safety factor, which allows assuming the design load corresponding to satisfactory reliability level, is calculated.

\section{PROBABILITY OF FAILURE AND FACTOR OF SAFETY}

The reliability theory is based on the concept of limit state function $f(\mathbf{X})$. This function, being dependent on the vector of random variables $\mathbf{X}$, is defined in such a way as to be greater or equal to zero for the safe states and less than zero for states of failure. In the case of bearing capacity of foundation the limit state function can be expressed as the difference of the load $\mathrm{L}$ and the bearing capacity $P$, i.e.,

$$
f(\mathbf{X})=P(\mathbf{X})-L(\mathbf{X}) .
$$

In the present paper, the load has been assumed as deterministic, independent of the vector of random variables.
The probability of failure can now be defined as

$$
p_{F}=p(f(\mathbf{X})<0)=p(P(\mathbf{X})<L)
$$

where $p(x)$ denotes probability of $x$. If joint probability density function for vector $\boldsymbol{X}$ (denoted as $p_{X}(\boldsymbol{X})$ ) is known equation (2) can be written as

$$
p_{F}=\int_{f(\mathbf{X})<0} p_{X}(\mathbf{X}) d \mathbf{X} .
$$

Because reliability theory usually takes interest in low probability values and the region of integration often has a specific shape in many cases some additional estimation techniques have to be used to evaluate integral (3). First Order Reliability Method, Second Order Reliability Method and Monte Carlo Simulation are particularly popular techniques. When limit state function is strongly nonlinear and there is a possibility of existence of multiple integration regions (the integration region is a disconnected set) the Monte Carlo Simulation method is usually the most reasonable choice (e.g., Gupta and Manohar [5]).

The concept of global factor of safety $F$ is usually associated with a simple measure of reliability, utilizing only the mean of random variable, i.e.,

$$
F=\frac{[P(\mathbf{X})]}{L}
$$

where $[x]$ denotes the mean of $x$. Approach (4) can be extended when searching, for instance, how many times the value of applied load should be greater than mean value of bearing capacity $P(\mathbf{X})$ in order to reduce the probability of failure to a given value. Definition (4) in such approach changes to:

$$
F=\frac{[P(\mathbf{X})]}{L}, \quad \int_{P(\mathbf{X})-a<0} p_{X}(\mathbf{X}) d \mathbf{X}=p_{D}
$$

where $p_{D}$ is the desired value of the probability of failure following a satisfactory level of the structure reliability, $a$ denotes the value of load corresponding to $p_{D}$ and mean $[P(\mathbf{X})]$ can be defined as

$$
[P(\mathbf{X})]=\int_{-\infty}^{\infty} P(\mathbf{X}) p_{\mathbf{X}}(\mathbf{X}) d \mathbf{X}
$$

Such an approach requires knowledge of both the joint probability density function $p_{x}(\mathbf{X})$ as well as the bearing capacity $P(\mathbf{X})$ as a function of random variables. The calculated safety factor allows however appropriate value of design load to be assumed which will reduce the probability of failure of the structure to a given value. This approach will be used in the present work. 


\section{DETRMINISTIC CALCULATIONS OF BEARING CAPACITY}

As has been stated in the first section in the case of materials with strength anisotropy orientation of material direction is a particularly important factor which can strongly affect, e.g., bearing capacity of footing located on such material. For the micro-layered soil which is a case of transversal isotropy in order to orient material direction in the $3 \mathrm{~d}$ problem space two independent parameters need to be specified. In most of the geological studies these are dip and azimuth angles. The definition of these angles (denoted as $\alpha$ and $\beta$, respectively) is presented in Fig. 1.

Dip and azimuth angles as other geotechnical parameters of the soil can be determined incorrectly, with some error, or correctly, but in terms of statistics - with given distribution, mean and variance. Since the paper focuses on uncertainties in orientation of material direction in anisotropic medium, orientation parameters $\alpha$ and $\beta$ have been assumed as the only random variables in the study. Hence vector $\mathbf{X}=\{\alpha, \beta\}$. The variables have been assumed as uncorrelated.

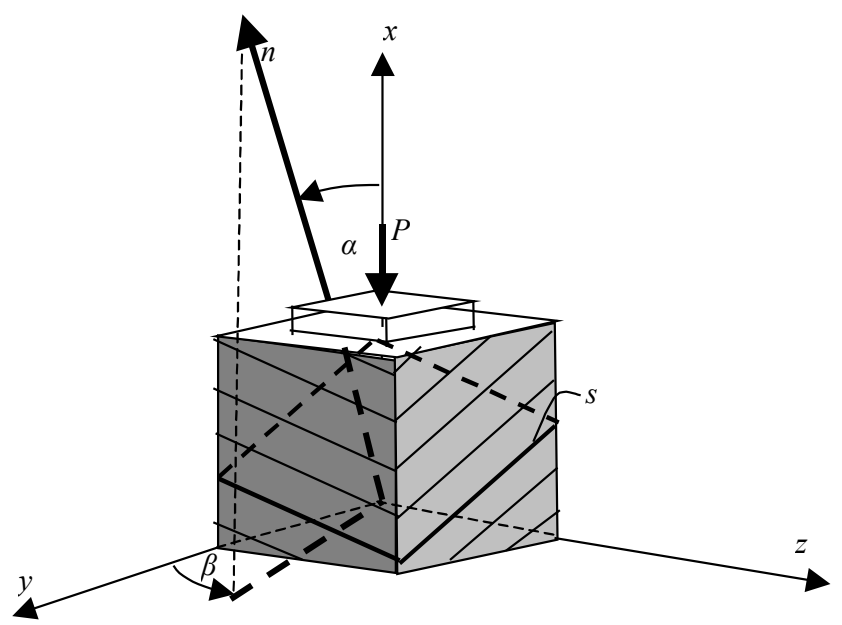

Fig 1. Square footing located on micro-layered soil in 3D. The orientation of lamination plane defined by angles $\alpha$ and $\beta$

The proposed methodology of safe design of the structure located in anisotropic soil involves calculation of global factor of safety (5). This factor is equal to the ratio between the value of applied load and the mean value of bearing capacity $P(\mathbf{X})$ which should be reached in order to reduce the probability to appropriate safety level. The safety factor defined in this way should be easy for application as additional safety factor for design load. As has been mentioned in the previous section calculation of the safety factor
(5) requires knowledge of the function $P(\mathbf{X})$, i.e., the bearing capacity as a function of random variables. The dependence of $P$ on angles $\alpha$ and $\beta$ is generally not known, however it can be approximated based on the results of calculation of $P$ obtained discretely for many different values of orientation angles.

A series of calculations of bearing capacity of square footing on micro-layered soil has been performed utilizing finite difference code FLAC3D [3]. Material of soil has been assumed to be elastic - perfectly plastic with associated flow rule and the phenomenological criterion presented in the work by Kawa and Łydżba [7] has been used as plasticity function. The criterion is a conjunction of two models: Pariseau anisotropic criterion [9]

$$
A_{i j}+\sqrt{\sigma_{i j} A_{i j k l} \sigma_{k l}}-1 \leq 0,
$$

and Jaeger critical plane equation (1960)

$$
|\tau|+\frac{3 a_{c} \sigma_{n}-k_{c}}{\sqrt{1-12 a_{c}^{2}}} \leq 0
$$

where $\tau, \sigma_{n}$ denote tangential and normal components of stress vector on weakness plane, $\sigma_{i j}$ is the stress tensor, $k_{c}$ and $a_{c}$ are strength parameters for weakness plane and $A_{i j}, A_{i j k l}$ are anisotropic strength tensors for matrix which can be expressed as

$$
\begin{gathered}
A_{i j}=\left[\begin{array}{ccc}
U & 0 & 0 \\
& V & 0 \\
s y m . & & V
\end{array}\right], \\
\\
A_{i j k l}
\end{gathered}
$$

In the work by Łydżba and Kawa [8] the criterion has been shown to be in a very good agreement with strength results obtained using homogenization technique for two-constituent micro-layered microstructure with strength of constituents ruled by the Drucker -Prager condition. The latter condition is usually presented as

$$
a I_{1}+\sqrt{J_{2}}-k \leq 0
$$

where $I_{1}$ denotes first invariant of the stress tensor, $J_{2}$ second invariant of the stress deviator, and $a$ and 


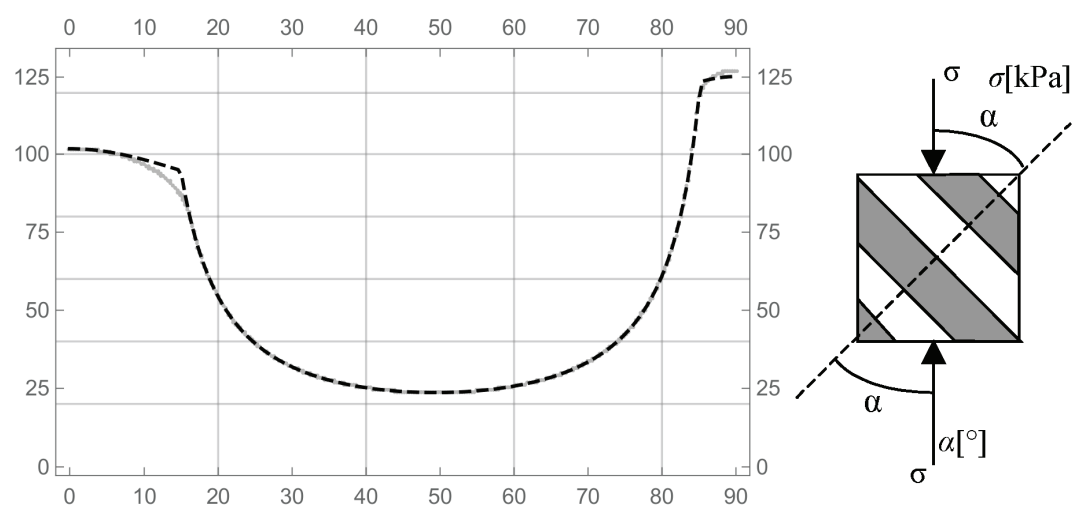

Fig. 2. Directional uniaxial compression strength of micro-layered soil considered. Comparison of results from micromechanics (gray line) with identified phenomenological model (dashed line)

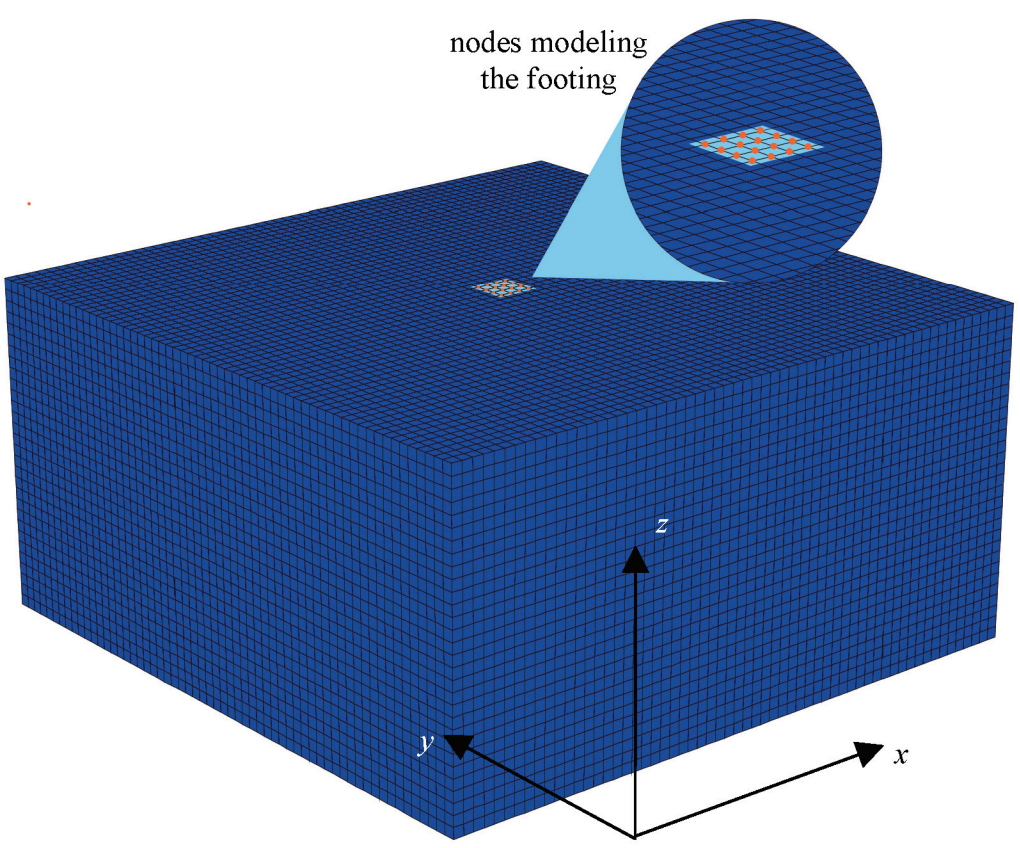

Fig. 3. The finite difference model for deterministic calculations

$k$ are strength parameters which, in simplification, correspond to friction and cohesion. The methodology of identification of all seven parameters of the proposed criterion (i.e., $a_{c}, k_{c}, U, V, G, F$ and $M$ ) based on strength properties and volume fraction of constituents for the two-constituent Drucker-Prager microstructure has also been presented in the work cited.

For the present study the criterion has been identified for two-constituent layer microstructure with DruckerPrager strength parameters of constituents equal to $a_{1}=$ $0.15, c_{1}=100 \mathrm{kPa}, a_{2}=0.05, c_{2}=10 \mathrm{kPa}$, respectively, and the equal volume fraction of both constituents. Following the methodology presented in the previous work (Kawa and Łydżba [7]) the values of parameters of the criterion have been identified as $U=2.44 \mathrm{e}-4, V=2.56 \mathrm{e}-3$, $G=5.08 \mathrm{e}-5, F=6.06 \mathrm{e}-5, M=3.01 \mathrm{e}-4, a_{c}=0.05$ and $k_{c}=10$. In Fig. 2, exemplary graph of directional uni- axial compression strength obtained for the microstructure considered with micromechanics method in comparison with results obtained for identified criterion has been presented. In can be seen both that the identified criterion is indeed in very good agreement with the failure function of the microstructure and that the microstructured soil considered is a strongly anisotropic medium.

In order to solve boundary value problem of bearing capacity in FLAC3D the micro-layered soil has been divided into $60 \times 60 \times 30$ uniform cubic zones modeling $15 \mathrm{~m} \times 15 \mathrm{~m} \times 7.5 \mathrm{~m}$ soil cuboid (Fig. 3). The external $y z$ planes, $x z$ planes and bottom $x y$ plane of the cuboid have been fixed in $x, y$ and $z$ directions, respectively. The square footing has been assumed as superficial, perfectly smooth with the dimensions $1 \mathrm{~m} \times 1 \mathrm{~m}$. It has been modelled with the 16 nodes for 


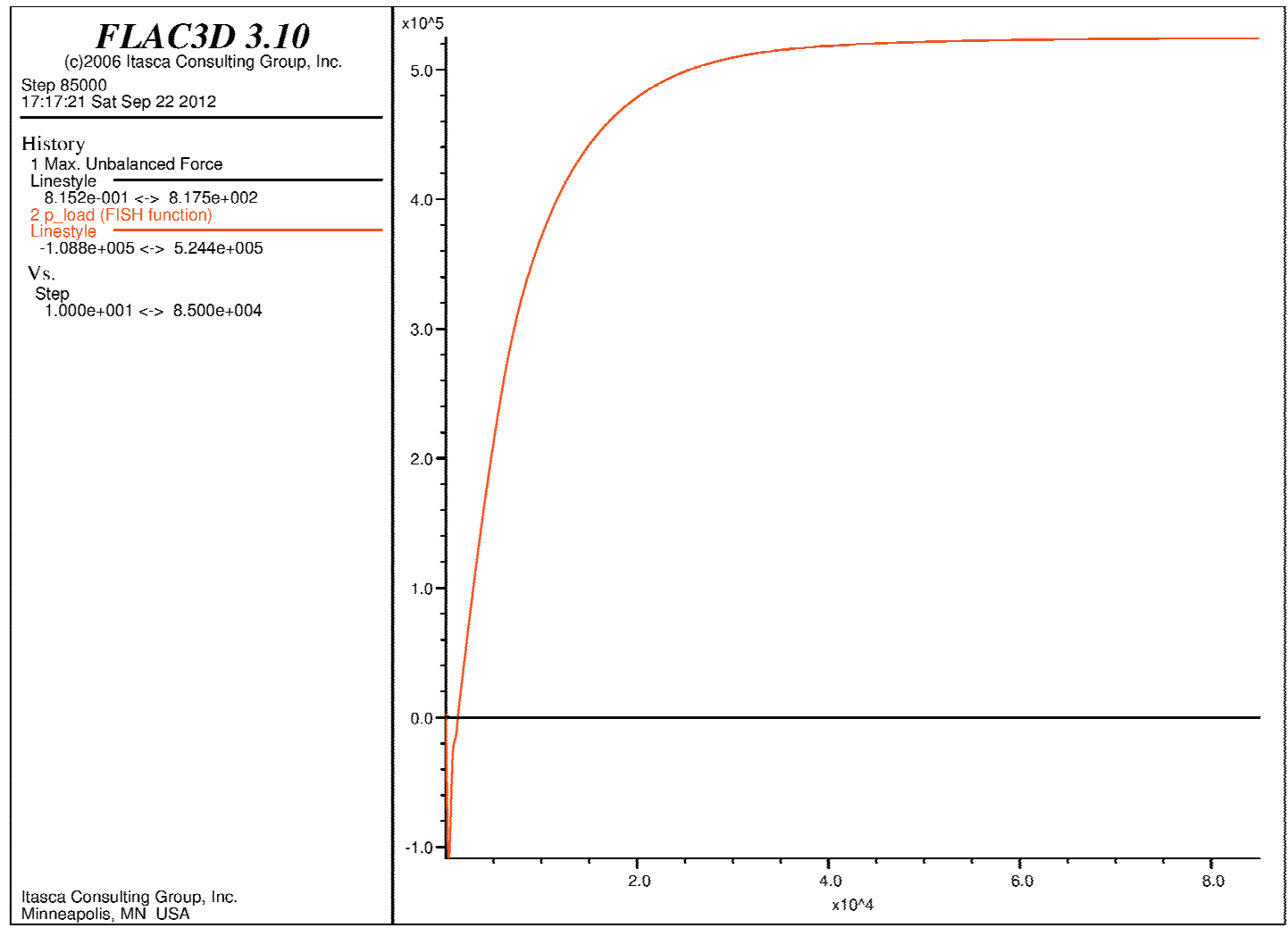

Fig. 4. Typical dependence of $P[N]$ on the number of time steps for single orientation

which constant velocity equal to $10^{-5} \mathrm{~m} /$ time step has been applied. The force $P$ has been calculated in each time step as a sum of the reactions obtained in these nodes. The calculations had been carried out until critical, constant value of $P$ was obtained. The typical dependence of the $P$ on the number of time steps is presented on Fig. 4. The calculations for single orientation took over 5 hours on standard PC.

Table 1 . Bearing capacity of square footing $P$ for different values of angles $\alpha$ and $\beta[\mathrm{MN}]$

\begin{tabular}{|c|c|c|c|c|c|}
\hline$\beta$ & $0^{\circ}$ & $22.5^{\circ}$ & $45^{\circ}$ & $67.5^{\circ}$ & $90^{\circ}$ \\
\hline $0^{\circ}$ & 0.4980 & 0.4321 & 0.4069 & 0.4478 & 0.5191 \\
\hline $22.5^{\circ}$ & 0.4980 & 0.4356 & 0.4137 & 0.4515 & 0.5287 \\
\hline $45^{\circ}$ & 0.4980 & 0.4378 & 0.4181 & 0.4531 & 0.5361 \\
\hline $67.5^{\circ}$ & 0.4980 & 0.4356 & 0.4137 & 0.4515 & 0.5287 \\
\hline $90^{\circ}$ & 0.4980 & 0.4326 & 0.4085 & 0.4490 & 0.5244 \\
\hline
\end{tabular}

The solutions obtained for values of orientation angles $\alpha$ and $\beta$ changing from 0 to $90^{\circ}$ by $22.5^{\circ}$ are presented in Table 1 and graphically in Fig. 5. As can be seen in Fig. 3 bearing capacity of footing $P$ is strongly nonlinear with respect to angle $\alpha$ and slightly nonlinear with respect to angle $\beta$. The presented result has been used to construct approximation of $P(\alpha, \beta)$ and calculate safety factor $F$.

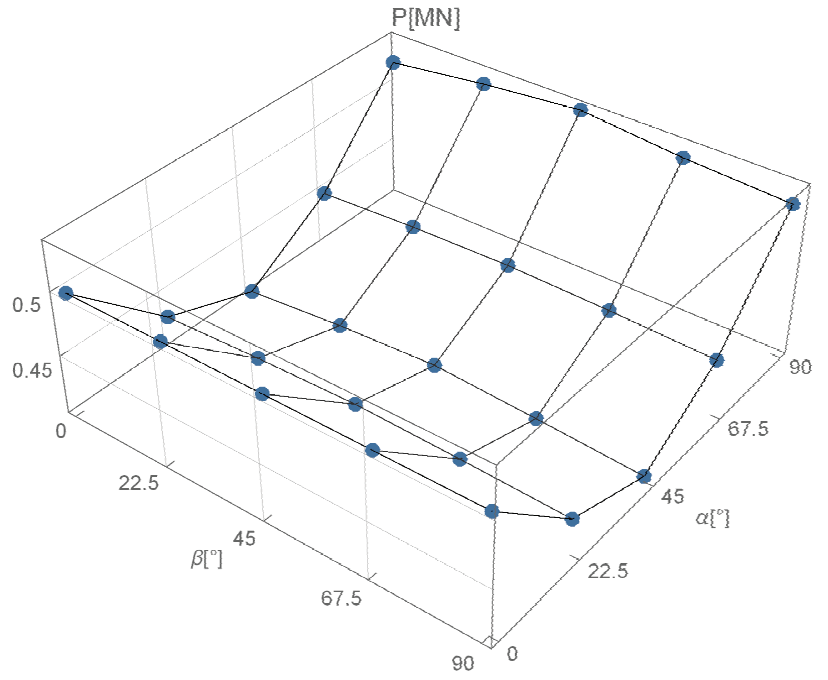

Fig. 5. Bearing capacity of square footing for different values of angles $\alpha$ and $\beta$

\section{APPROXIMATION OF FUNCTION OF BEARING CAPACITY}

In order to evaluate failure probability and global safety factor, explicit form of function $P(\alpha, \beta)$ is needed. The values of bearing capacity $P$ can be obtained only numerically and no explicit form of the 
function is given. In order to approximate function $P(\alpha, \beta)$ based on results presented above the Response Surface Method has been used.

Response Surface Method (e.g., Faravelli [4]) is a method particularly popular for its application to structural reliability analysis. The main goal of this method is to approximate unknown function using its known values obtained in a set of experimental points. Polynomials of order two, often without mixed terms, are usually chosen as a class of approximation function. In the problem considered, taking into account its symmetries and periodicity with respect to both random variables, a trigonometric function of the form

$$
\begin{gathered}
P(\alpha, \beta)=a+b \cos (2 \alpha) \\
+c \cos (2 \beta)+d \cos ^{2}(2 \alpha)+e \cos ^{2}(2 \beta)
\end{gathered}
$$

has been chosen as adequate.

Parameters of approximation function are usually obtained using normal regression with least square method. That means that all experimental point values are treated as equally important. It is worth noting that in formula (3) function $P(\alpha, \beta)$ as a part of limit state function influences only the region of integration. The closure of this region is a set of points where limit state function $f(\alpha, \beta)$ is equal to 0 . For evaluation of probability of failure $p_{F}$ function $P$ needs to be then approximated precisely only in the neighbourhood of this closure. The approximation should be then exceptionally good at experimental points which are located closest to curve $f(\alpha, \beta)=0$. For evaluation of $p_{F}$ parameters of approximation function should be therefore obtained using the weighted regression method rather than normal regression.

The weighted regression procedure for identifying the response surface in order to evaluate failure probability has been presented in work by Kaymaz and McMahon [10]. The proposed procedure has two steps. In the first step, normal regression is used to obtain basic approximation of the function. Then the importance of sample points is tested. Sample point for which absolute value of approximated limit state function is closest to zero is chosen as most important

$$
\hat{y}_{\text {best }}=\min |f(\mathbf{X})|
$$

and weights for all sampling points are evaluated as

$$
w_{i}=\exp \left(-\frac{\left|f\left(x_{i}\right)-\hat{y}_{\text {best }}\right|}{y_{\text {rel }}}\right)
$$

Subsequently updated version of approximation is evaluated utilizing weighted regression with weights (13).
In the original method by Kaymaz and McMahon the value $y_{\text {rel }}$ which is denominator in expression (13) is taken as equal to (12). It often happens that value (12) is very small and the results of weighted regression with that assumption become unreasonable. Taking into account the scale of the problem and maximal differences in values of function $P$ the value 0.01 has been assumed as a reasonable one for $y_{\text {rel }}$. This value has been used in the present calculations.

It should be noted that the above two-steps procedure can be useful for evaluation of $p_{F}$ (3) (or $F(5)$ ) but not the mean value of $P(6)$. In the latter case, value of integral depends on values obtained for all sample points which belong to the domain considered. For that purpose normal regression should be used, at least in the case of uniform distribution assumed for random variables in the domain.

\section{CALCULATION OF FACTOR OF SAFETY}

In order to show the procedure for calculating the factor of safety two different examples have been chosen. In the first case it is assumed that no information about lamination direction for micro-layered soil considered has been provided. The probability of failure of footing is therefore calculated under assumption that all possible directions of lamination are equally probable. A uniform distribution over the whole interval from 0 to $90^{\circ}$ is assumed for both orientation angles (Fig. 6). For the second example the assumptions are completely different: for both orientation angles full statistical information, i.e., distribution, mean and variance, is specified. The triangular distribution has been assumed as appropriate for that case. In both examples the required level of probability of failure has been assumed to be equal to 0.02 .

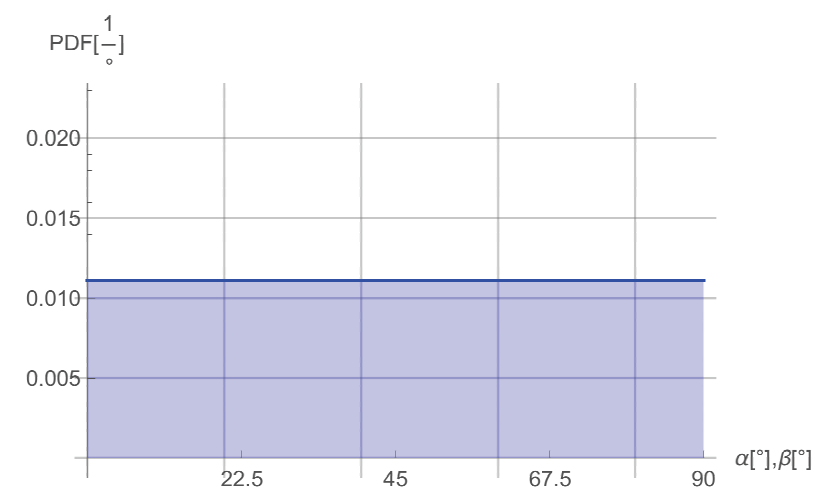

Fig. 6. The Probability Density Functions for orientation angles used in the first example 


\subsection{UNIFORM DISTRIBUTION FOR BOTH ORIENTATION ANGLES}

In the first case the approximation of function $P(\alpha, \beta)$ has been initially obtained using normal regression, with equal weights for every sample point. The general form of approximation has been chosen as (11). The obtained values of parameters are $a=0.405, b=$ $-1.35 \mathrm{e}-2, c=-5.73 \mathrm{~d}-4, d=0.106, e=7.09 \mathrm{e}-3$.

The obtained approximation has been presented in Fig. 7. It can be seen that the function has lower values than sample points obtained for $\alpha=45^{\circ}$. For the points denoted in Fig. 7 as $1-5$ the differences between values obtained deterministically and value of approximation function are $-9.43 \mathrm{e}-3,-1.25 \mathrm{e}-2$, $-1.30 \mathrm{e}-2,-1.17 \mathrm{e}-2,-9.88 \mathrm{e}-3$, respectively.

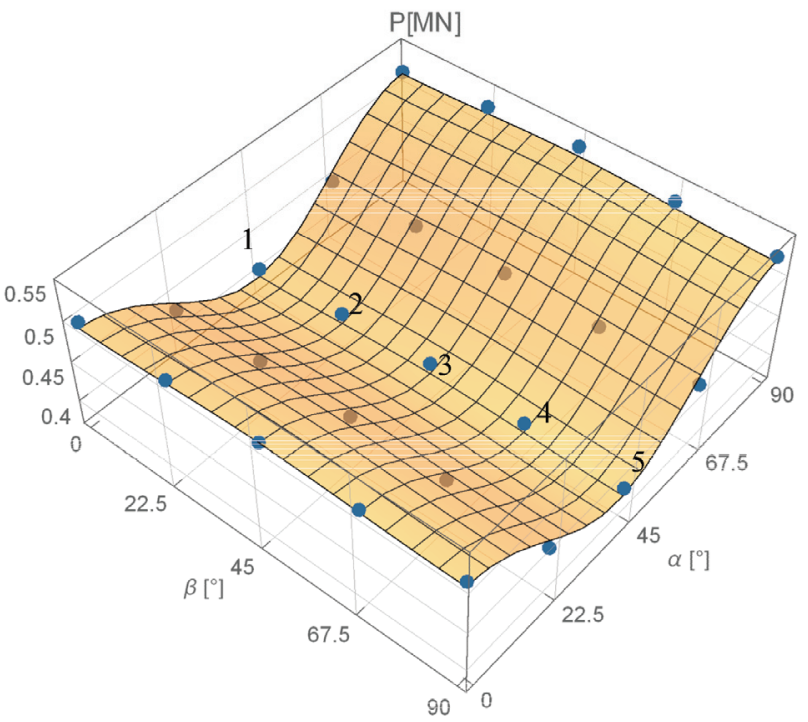

Fig. 7. First step of approximation of function $P(\alpha, \beta)$

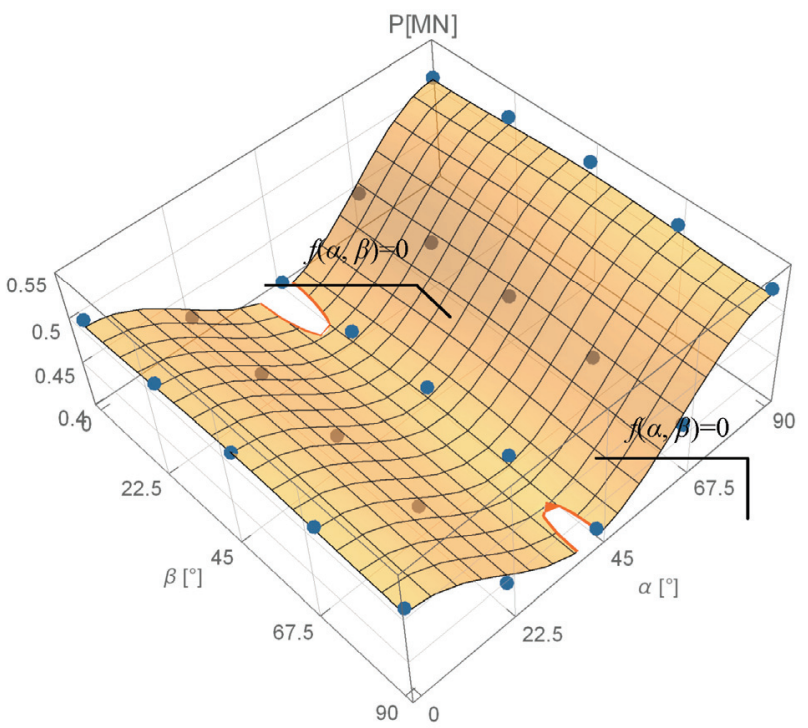

Fig. 8. Function from Fig. 3 with exclusion of the regions of failure
Using the presented approximation the probability of failure (3) has been calculated for different values of $L$. Numerical calculation of $p_{F}$ has been performed utilizing Monte Carlo Simulation with $10^{6}$ realizations within the COMREL package (www.strurel.de). The given level of failure probability 0.02 has been reached for $L=0.3993 \mathrm{MN}$.

In Fig. 8, the approximation of function $P(\alpha, \beta)$ have been once again presented. This time the regions where limit state function takes values lower than zero (for obtained value of $L=0.3993$ ) has been excluded from the diagram. It can be seen that curve $f(\alpha, \beta)=0$ which is a closure of excluded region is located close to some of experimental points obtained for $\alpha=45^{\circ}$. Seeing this it can be concluded that absolute value of limit state function (12) should be minimal for one of these points.

Using the procedure by Kaymaz and McMahon [10] an updated version of approximation function has been obtained (Fig. 9). The values of coefficients obtained with weighted regression are $a=0.419, b=$ $-1.35 \mathrm{e}-2, c=-5.73 \mathrm{~d}-4, d=6.31 \mathrm{e}-2, e=-1.08 \mathrm{e}-2$. This time the approximated function is in better agreement with values of experimental points $1-5$ : the differences between the approximation and the experimental points are now equal to $3.74 \mathrm{e}-4,-8.52 \mathrm{e}-4$, $5.64 \mathrm{e}-4,-2.26 \mathrm{e}-5,-5.28 \mathrm{e}-5$, respectively, i.e., about two orders smaller than using normal regression. The value of load for which the probability of failure reaches 0.02 is greater: $L=0.409 \mathrm{MN}$.

In order to evaluate a global factor of safety a mean value of $P$ needs to be known. This value has been evaluated by application of formula (6) to the approximation function obtained with normal regression (Fig. 9). The value of $[P(\alpha, \beta)]$ has been obtained as 0.4561 . The global factor of safety (5) has been calculated as equal to 1.11 .

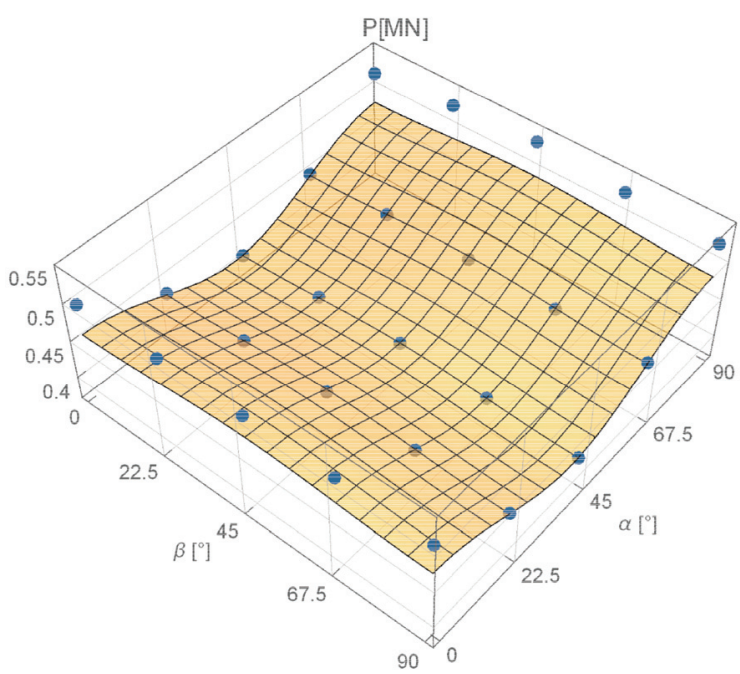

Fig. 9. Updated approximation of $P(\alpha, \beta)$ obtained using procedure by Kaymaz and McMahon [10] 


\subsection{TRIANGULAR DISTRIBUTION FOR BOTH ORIENTATION ANGLES}

Using similar numerical procedure as described above global safety factors have been evaluated also for the second numerical example. In that case both orientation angles $\alpha$ and $\beta$ have been assumed to have triangular distributions presented in Fig. 10. The range of variation of angle $\beta$ has been assumed to be from $22.5^{\circ}$ to $45^{\circ}$ with the mean value at $33.75^{\circ}$. Nine different distributions have been assumed for angle $\alpha$ with

$$
w_{i}=\exp \left(-\frac{\left\|x_{\text {peak }}-x_{i}\right\|}{d_{\text {rel }}}\right)
$$

where $\|x-y\|$ is a norm defining the distance between points $x$ and $y$, and $d_{r e l}$ denotes some constant comparative distance. Taking into account specific shape of probability density function the norm in formula (14) has been assumed as not Euclidean but

$$
\left\|x_{\text {peak }}-x_{i}\right\|=\max \left\{\left|\alpha_{\text {peak }}-\alpha_{1}\right|,\left|\beta_{\text {peak }}-\beta_{i}\right|\right\}
$$
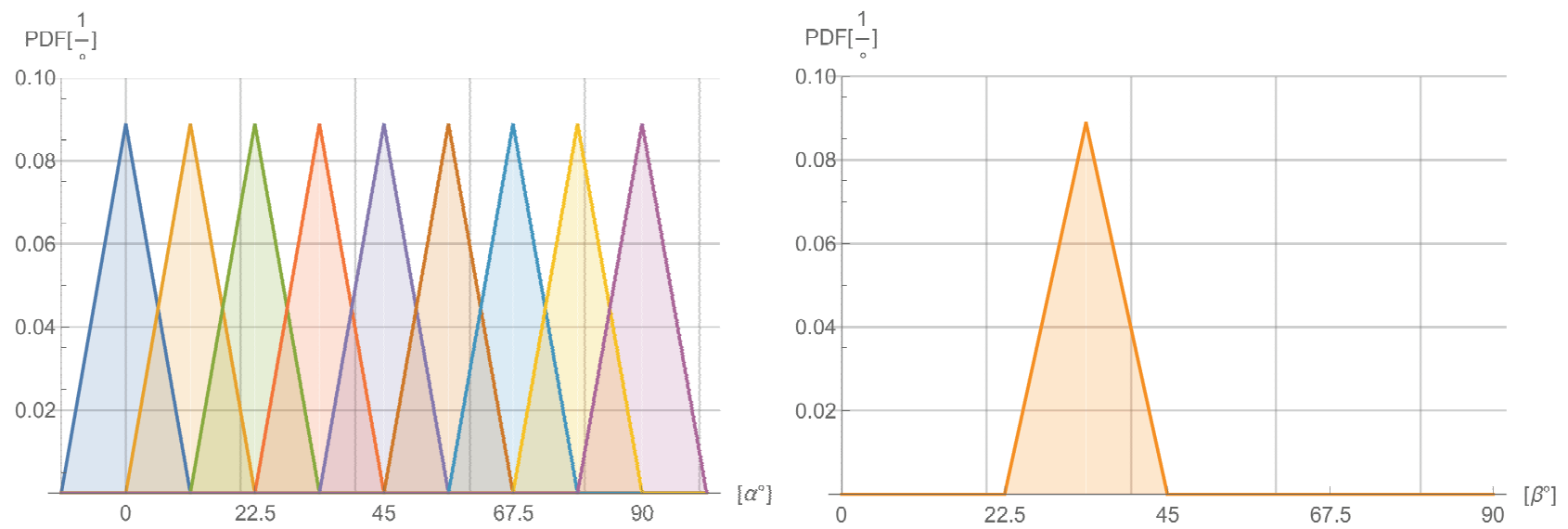

Fig. 10. Nine different distributions assumed for angle $\alpha$ and distribution assumed for angle $\beta$

the same variation range but with different mean values. Range of variation has been assumed identically as for $\beta$ as $22.5^{\circ}$, with the mean values equal to $0^{\circ}$, $11.25^{\circ}, 22.5^{\circ}, 33.75^{\circ}, 45^{\circ}, 57.25^{\circ} 67.5^{\circ}, 78.75^{\circ}, 90^{\circ}$, respectively. The value of global factor of safety has been calculated for all nine cases.

Note that this time, unlike in the first example, in approximation of function $P(\alpha, \beta)$ used to determine the mean value of bearing capacity (6), not all the experimental points should have the same weight. This is due to the fact that the applied probability distribution for random variables is not a uniform one. In consequence, the experimental points with higher values of probability density function are more important for the mean value of $P(X)$. The approximation at these points should be particularly good.

In order to improve the quality of the approximation used to determine the mean value of $P$ the weighted regression similar to the method by Kaymaz and McMahon [10] has been used. The main difference is that the weights for the specific points depend not on the value of limit state function but on the distance of considered point from the point with highest value of probability density function $x_{\text {peak }}$. The weights can be then expressed as and the comparative distance $d_{r e l}$ has been assumed as equal to half of variability range, i.e., $11.25^{\circ}$.

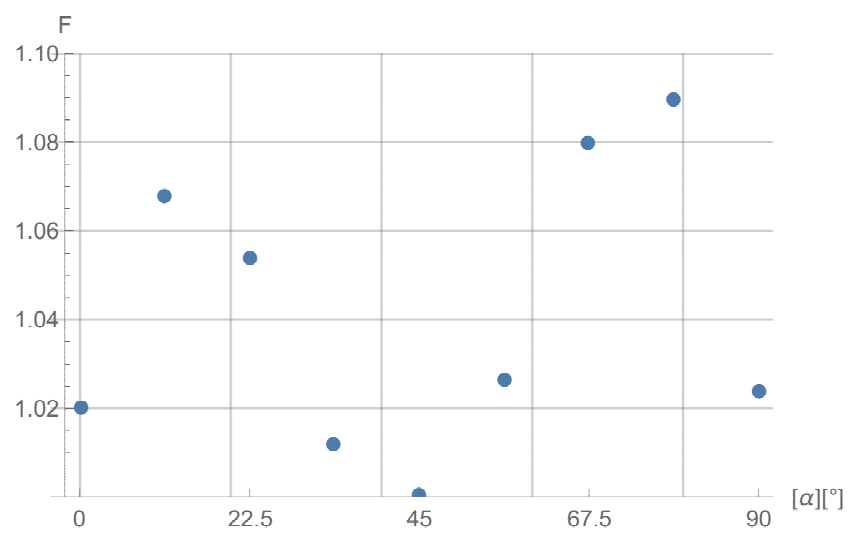

Fig. 11. Global factor of safety for different mean values of angle $\alpha$

The approximation of function $P$ used to calculate its mean value for (6) has been obtained in one step using weighted regression with the weights (14). All the previous calculation steps regarding the failure probability as well as final calculation of the 
factor of safety are preformed identically as in the first example.

The factor of safety obtained for 9 cases of distribution assumed for angle $\alpha$ is presented in Fig. 11 . It can be seen that global factor of safety varies from slightly over 1.00 to 1.09 and is smaller than the factor obtained in the first example. This shows both that the strength anisotropy influences the value of global factor of safety and that providing more information about the orientation of lamination direction should result in a smaller value of safety factor.

\section{CONLUSIONS}

In the present paper, reliability analysis of bearing capacity of square footing on soil with strength anisotropy due to layered microstructure has been conducted. The random variables involved in the analysis are two angles orienting lamination direction. The probabilistic modelling has been based on boundary value problem solution obtained with deterministic calculation for a variety of orientations. Using these results the anisotropic function of bearing capacity has been approximated with Response Surface Method utilizing weighted regression proposed by Kaymaz and McMahon [10]. As a result of analysis for two different cases the procedure for calculating a global factor of safety for a given probability of failure has been shown. The obtained values indicate how many times the design load has to be greater than the mean value of bearing capacity of footing to reduce the probability of failure to a given value.

The following conclusions can be drawn from the study:

(i) The presented procedure allows to one obtain value of the factor of safety. This factor can be applied as additional safety factor for analysis involving anisotropic medium. As typically in the case of using Response Surface Method the greatest disadvantage of the procedure is great amount of time consumed by deterministic analysis.

(ii) The values of safety factor obtained for the second example when more statistical information has been provided are smaller than in the first example. This fact follows general intuition: the more statistical information is available, the more risk of failure can be reduced. This also proves that providing more statistical information results in more economic design.
(iii)As can be seen in the second example the mean value of dip angle influences the value of the safety factor. Comparing Fig. 11 and Fig. 5 it is possible to conclude that the maximal values of safety factor correspond to the ranges of orientation angles where bearing capacity function changes most dynamically. Using this dependence, when some knowledge about the possible values of dip and azimuth angle as well as bearing capacity function is given, the more and less risky situations are possible to be distinguished even without detailed calculations.

(iv)All the obtained values of global factor of safety are relatively small which means that in the case considered wrong estimation of lamination direction has little effect on the solution and the reliability of the structure. For some other cases the influence of anisotropy of the solution of boundary value problem can be much greater. In this context the presented approach can usefully combine the precise numerical calculations incorporating anisotropic model together with reliability tools to ensure safety and economy of construction.

The presented procedure is subjected to one important simplification which needs to be taken into account when applying the method for designing real constructions. The simplification is based on the assumption of probability of failure being equal to 0.02 . In terms of reliably theory it is a rather big value. For typical long-term constructions European code of practice (EC0) suggests the value of reliability index to be equal 3.8 which corresponds to the probability of failure equal to $7 \mathrm{e}-5$. Changing the assumed probability value from 0.02 to $7 \mathrm{e}-5$ will not affect the presented procedure as well as it should not strongly affect the calculated values of safety factor. However, to ensure the accuracy of calculation of integral (3) the approximation of bearing capacity function needs to be improved. That means both: the number of experimental points should be significantly increased and more accurate approximation method, perhaps a multi-step procedure, should be used. This is a subject of further studies of the author.

\section{REFERENCES}

[1] BRZĄKAŁA W., Strength modelling of a randomly fissured material, Studia Geotechnica et Mechanica, 2005, Vol. 27, No. 1-2, 33-41.

[2] Duveau G., Shao J.F., Henry J.P., Assessment of some failure criteria for strongly anisotropic rocks, International Journal for Numerical and Analytical Methods in Geomechanics, 1997, Vol. 1, 1-26, John Wiley \& Sons. 
[3] FLAC3D (2007) Fast Lagrangian Analysis of Continua. Itasca.

[4] FARAVELLI L.A., A response surface approach for reliability analysis, Journal of the Engineering Division, ASCE, 1989, 115 (12), 2763-2781.

[5] Gupta S., Manohar C.S., An improved response surface method for the determination of failure probability and importance measures, Structural Safety, 2004, 26, 123-139.

[6] JAEGER J.C., Shear failure of anisotropic rocks, Geological Magazine, 1960, Vol. 27, No. 1.

[7] KAWA M., ŁYDŻBA D., Strength criterion for geomaterials with layered microstructure, Górnictwo i Geoinżynieria, 2008, z. 2 (in Polish).
[8] ŁydżBA D., KAwA M., Failure mechanism of sedimentary rocks: micromechnics approach, [in:] 2nd International Symposium on Computational Geomechanics (COMGEO II), Cavtat-Dubrovnik, April 2011, pp. 27-29.

[9] PARISEAU W.G., Plasticity theory for anisotropic rocks and soils, Proceedings of 10th Symposium on Rock Mechanics (AIME), 1972.

[10] Kaymaz I. MCMAHON C., A response surface method based on weighted regression for structural reliability analysis, Probabilistic Engineering Mechanics, 2005, 20, 11-17.

[11] Vessia G., Cherubini C., PieczyŃSKa J., PUŁa W., Application of random finite element method to bearing capacity design of strip footing, Journal of Geoingineering, 2009, Vol. 4, No. 3. 